\title{
Preferential treatment in procurement auctions through information revelation
}

\author{
Domenico Colucci*, Nicola Doni ${ }^{\dagger}$ and Vincenzo Valori ${ }^{\ddagger}$
}

July 1st, 2012

\begin{abstract}
We study a model of procurement auctions in which information policies can be used to treat two heterogeneous suppliers asymmetrically. The buyer is shown to be better off revealing information about her preferences to the weak supplier only, when there is a sufficient cost difference between the weak and the strong. Conversely, when the two competitors have similar cost structures, for the buyer it is best to disclose her preferences publicly.

JEL: D44, D82, H57

Keywords: procurement, information revelation, discriminatory policy, asymmetric auctions
\end{abstract}

\section{Introduction}

There is a wide debate and a correspondingly large literature about preferential treatment in public procurement auctions. It has been documented that allowing for bid preferences of disadvantaged bidders may in some cases reduce procurement costs (see e.g. McAfee and McMillan [10], Rothkopf et al. [11], Hubbard and Paarsch [4], Corns and Schotter [2]). Granting a right of first refusal (see e.g. Lee [7]) is also a way of implementing discriminatory treatment in procurement.

In this paper we enquire whether similar effects can be obtained by giving informational advantages. In particular we study the case in which a buyer keeps into account non-price attributes when procuring goods and services. There are situations in which such attributes are a function of both the buyer's preferences and demands on one side and the objective properties of the suppliers on the other. For example consider a government agency in need of computer equipment: suppose the agency wants to procure the equipment for either its legal branch or its IT department; its needs in the two cases are bound to differ considerably. In any case the agency knows what it is best for its purposes. In turn the supplier may be more or less focused or specialized on some or other aspect

\footnotetext{
*Università di Firenze, Dipartimento di Matematica per le Decisioni, domenico.colucci@unifi.it.

${ }^{\dagger}$ Università di Firenze, Dipartimento di Scienze Economiche, nicola.doni@unifi.it.

‡Università di Firenze, Dipartimento di Matematica per le Decisioni, vincenzo.valori@unifi.it.
} 
(computational power, durability, GPU speed etc.). How much the goods produced by a given supplier fit the buyer's needs is the outcome of various factors pertaining to both parties (see also Gal-Or et al. [3]). Here we model this situation by assuming that the quality assessment of each of two potential suppliers is determined as the result of the match between buyer's preferences and the supplier's technical characteristics: information about qualities hence depends on the extent to which such characteristics are known to both competitors. One possibility is that both are public, which refers to the case in which both firms are well-established and vie for the procurement contract. At the opposite end there is the case in which none of the two characteristics are known, as in markets for new products or technologies. There is finally the possibility that only one of the two suppliers' characteristics are publicly known (e.g. the incumbent in a long going relation with the buyer). At the same time a supplier needs to know the buyer's preferences to figure out the qualities. So the bidders actual information sets depend on whether the buyer decides to reveal her preferences and to whom, i.e. on her information policy. Finally the two suppliers are assumed to differ in terms of costs (which can model the idea of a local firm facing strong competition from an aggressive foreign firm). We compute the expected return for the bid-taker as a result of her information policy and the context.

This paper's contribution is to give conditions that make it beneficial for the buyer to reveal information to the weak supplier only: such preferential treatment is optimal when there is a sufficient cost difference between the weak and the strong (with precise details depending on the specific information architecture). Conversely, when the two competitors are similar, for the buyer it is best to disclose her preferences publicly. This result is in line with the above mentioned literature, in particular it is close in spirit to the conclusions of Lee $[7]$.

The scope of the paper is limited by the introduction, for tractability reasons, of some technical assumptions: the model has two bidders and uses the uniform distribution (see e.g. Lee [7], Kim [6] for analogous choices in related models).

\section{Model}

Two risk-neutral suppliers with different, commonly known production costs compete to provide a good or service; they are labelled $w$ and $s$ (with costs $c_{w}, c_{s}$ with $c_{w}-c_{s} \equiv \Delta>0$ ). Each tenders a bid, comprising a price $p_{i}$ (for $i=w, s$ ) and technical specifications (which we assume to be exogenously given), with the aim of maximizing his expected profit. The buyer cares for non-price attributes, modelled here as quality, which she assigns to the specific product provided by each bidder, given the suppliers' technical characteristics and her own specific preferences. She specifies before the auction an information policy, which may entail concealment or public revelation of her preferences (symmetric policies), or she may reveal preferentially to only either $w$ or $s$ (asymmetric policies). Hence we consider the four policies $C$ (concealment), $P U$ (public), $A S_{w}$ (revelation to the weak) and $A S_{s}$ (revelation to the strong).

The quality parameters $q_{i}$ are uncertain unless one knows both characteristics and preferences: short of such knowledge players believe qualities to be generated by independent draws from the uniform distribution on the unit interval. The buyer awards the contract to the supplier providing the largest quality 
minus price difference, $q_{i}-p_{i}$ (labelled score henceforth). ${ }^{1}$ The winner earns a profit of $p_{i}-c_{i}$.

There are four possibilities as regards the information on characteristics: they may be common knowledge or they may be privately known by either both or one (the weak or the strong) of the bidders: so we distinguish four scenarios accordingly. In each, the specifics of the actual information available to the suppliers depend on what they know about their competitor and on whether the buyer's policy endows them of knowledge on her preferences.

\section{Bidders' equilibrium strategies}

Each supplier's problem is to maximize his expected net profit given the information available to him. Such information sets may comprise knowledge of both qualities, of only one's own quality or none of those. Formally, $i$ 's information set may be one of $\left\{q_{i}, q_{j}\right\},\left\{q_{i}\right\}, \varnothing$ and it is determined by the information setup on suppliers' characteristics and the information policy. So if preferences are revealed to $i$ and $i$ knows $j$ 's characteristics then $i$ 's information will be $\left\{q_{i}, q_{j}\right\}$, while it will be $\left\{q_{i}\right\}$ when $i$ ignores $j$ 's characteristics; it will be $\varnothing$ if preferences are not revealed.

When a supplier has both relevant pieces of information he is able to exactly match his competitor's score: indeed in equilibrium his bid will ensure such match (provided the bid covers his cost). ${ }^{2}$ If he can only rely upon one piece of information then he will resort to the probability distribution of his competitor's quality to assess his probability of winning the auction. If he knows none of the qualities he will evaluate such probability using the distribution of $q_{i}-q_{j}$.

\subsection{Suppliers' characteristics are public}

The four information policies available imply in this case the information sets for the bidders are as in the following table:

\begin{tabular}{|c|c|c|c|c|}
\hline & \multicolumn{2}{|c|}{ Symmetric policies } & \multicolumn{2}{c|}{ Asymmetric policies } \\
\hline & $C$ & $P U$ & $A S_{w}$ & $A S_{s}$ \\
\hline$I_{w}$ & $\varnothing$ & $\left\{q_{w}, q_{s}\right\}$ & $\left\{q_{w}, q_{s}\right\}$ & $\varnothing$ \\
\hline$I_{s}$ & $\varnothing$ & $\left\{q_{w}, q_{s}\right\}$ & $\varnothing$ & $\left\{q_{w}, q_{s}\right\}$ \\
\hline
\end{tabular}

Given the suppliers' characteristics are common knowledge, information on the bid-taker's preferences implies complete knowledge of qualities. In $C$ the equilibrium will be at the intersection of the best response functions $p_{i}\left(p_{j}\right)=$ $\arg \max \left(p_{i}-c_{i}\right) \operatorname{Pr}\left\{q_{i}-q_{j} \geq p_{i}-p_{j}\right\}$ and the symmetric $p_{j}\left(p_{i}\right)$ : this is an extension to asymmetric bidders of results from Gal-Or et al. [3]. In $A S_{s}$ the weak knows he will win only if his score is not feasible for his opponent: in other

\footnotetext{
${ }^{1}$ Notice that the score might be negative. One can restate the model to have qualities that range in an interval $[\underline{q}, \bar{q}]$, for a sufficiently high $\underline{q}$ so that the buyer always gets a positive utility from the auction. The results do not change however, so we stick to the simpler case of $\underline{q}=0, \bar{q}=1$.

${ }^{2}$ Define the tie breaking rule as follows. In case of equal score the winner will be the most informed supplier, which is akin to awarding a right of first refusal to the supplier with the richest information set. If the information sets have the same cardinality the tie is broken in favour of the supplier with the largest $q_{i}-c_{i}$ difference, which corresponds to a Vickrey rule.
} 
words he will bet on obtaining a score which the strong could only beat with a bid that implies a sure loss, so $p_{w}=\arg \max \left(p_{w}-c_{w}\right) \operatorname{Pr}\left\{q_{w}-q_{s} \geq p_{w}-c_{s}\right\}$ while $p_{s}=\max \left\{q_{s}-q_{w}+p_{w}, c_{s}\right\}$. The opposite case $A S_{w}$ is similar. In $P U$ we have $p_{i}=\max \left\{q_{i}-q_{j}+c_{j}, c_{i}\right\}$, i.e. a supplier matches his opponent's score whenever possible, so the winner is the one with the largest quality minus cost difference, $q_{i}-c_{i}$.

\subsection{Suppliers' characteristics are private}

In this case suppliers can at most get to know their quality but not that of their competitor. We have:

\begin{tabular}{|c|c|c|c|c|}
\hline & \multicolumn{2}{|c|}{ Symmetric policies } & \multicolumn{2}{c|}{ Asymmetric policies } \\
\hline & $C$ & $P U$ & $A S_{w}$ & $A S_{s}$ \\
\hline$I_{w}$ & $\varnothing$ & $\left\{q_{w}\right\}$ & $\left\{q_{w}\right\}$ & $\varnothing$ \\
\hline$I_{s}$ & $\varnothing$ & $\left\{q_{s}\right\}$ & $\varnothing$ & $\left\{q_{s}\right\}$ \\
\hline
\end{tabular}

So suppliers can at most get to know their own quality. In $P U$ the game can be shown to be equivalent to an asymmetric auction, in which bidders choose a score given a privately known valuation defined as $q_{i}-c_{i}$ (whose distributions are horizontally shifted by $\Delta$ ). So while closed form solutions, even with the uniform distribution, are not readily usable (see Maskin and Riley [9], Kaplan and Zamir $[5]$ ) we computed the buyer's expected utility by means of the BIDCOMP ${ }^{2}$ software (see Li and Riley [8]). In $A S_{w}$ the weak's equilibrium bid depends on $c_{w}, c_{s}$ and $q_{w}$ while the strong's is a function of the costs only (the actual functions are derived in a way similar to the $C$ case). The converse happens with $A S_{s}$.

\subsection{The weak's characteristics are public, the strong's are private}

If the strong supplier has the advantage of being the only one to know his characteristics we have:

\begin{tabular}{|c|c|c|c|c|}
\hline & \multicolumn{2}{|c|}{ Symmetric policies } & \multicolumn{2}{c|}{ Asymmetric policies } \\
\hline & $C$ & $P U$ & $A S_{w}$ & $A S_{s}$ \\
\hline$I_{w}$ & $\varnothing$ & $\left\{q_{w}\right\}$ & $\left\{q_{w}\right\}$ & $\varnothing$ \\
\hline$I_{s}$ & $\varnothing$ & $\left\{q_{w}, q_{s}\right\}$ & $\varnothing$ & $\left\{q_{w}, q_{s}\right\}$ \\
\hline
\end{tabular}

The $P U$ policy in this case implies that the strong will match his opponent's score whenever feasible, while the weak can only win if his quality compensates for the cost difference. Hence $p_{w}=\arg \max \left(p_{w}-c_{w}\right) \operatorname{Pr}\left\{q_{s} \leq q_{w}-p_{w}+c_{s}\right\}=$ $\max \left\{\frac{q_{w}+c_{w}+c_{s}}{2}, c_{w}\right\}$ and $p_{s}=\max \left\{q_{s}-q_{w}+p_{w}, c_{s}\right\}$.

\subsection{The weak's characteristics are private, the strong's are public}

Finally when the strong has the disadvantage of having his characteristics known we have: 


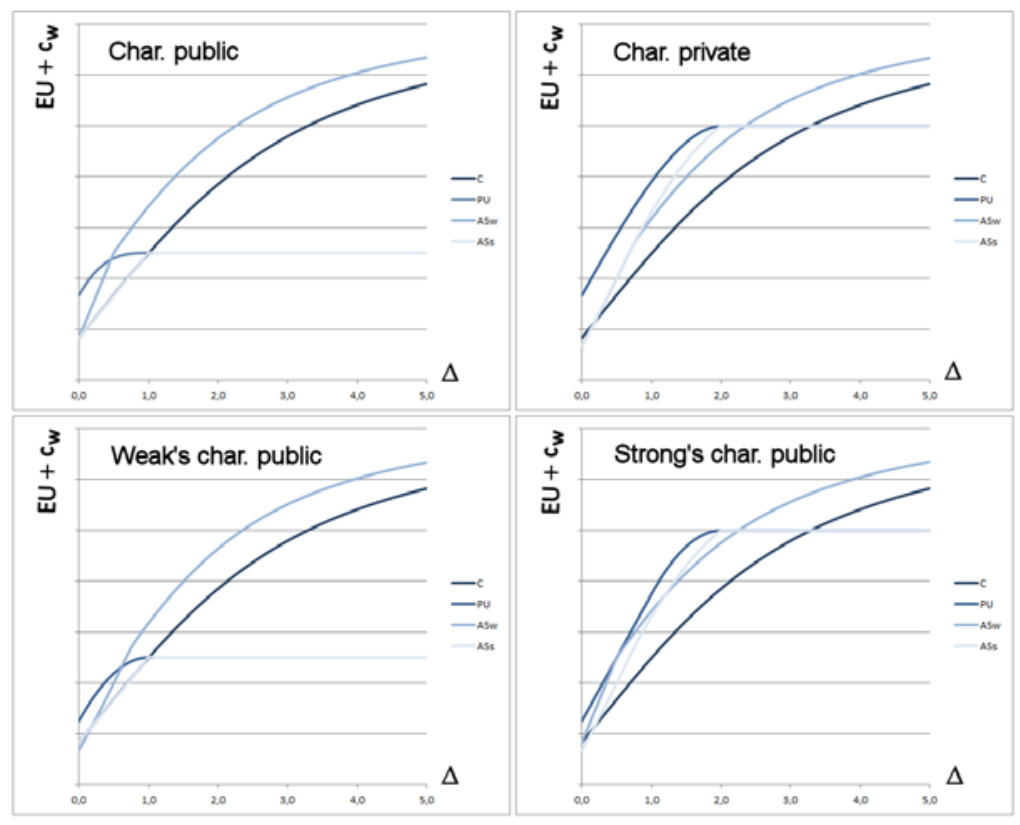

Figure 1: The buyer's expected utility in the four scenarios.

\begin{tabular}{|c|c|c|c|c|}
\hline & \multicolumn{2}{|c|}{ Symmetric policies } & \multicolumn{2}{c|}{ Asymmetric policies } \\
\hline & $C$ & $P U$ & $A S_{w}$ & $A S_{s}$ \\
\hline$I_{w}$ & $\varnothing$ & $\left\{q_{w}, q_{s}\right\}$ & $\left\{q_{w}, q_{s}\right\}$ & $\varnothing$ \\
\hline$I_{s}$ & $\varnothing$ & $\left\{q_{s}\right\}$ & $\varnothing$ & $\left\{q_{s}\right\}$ \\
\hline
\end{tabular}

The $P U$ policy in this case gives the weak full information which he'll use to match the other's score, if possible. The strong instead, given his cost advantage, uses the information on his own quality to either compete (even if $q_{s}$ is very low he might still win) or win for sure (if $q_{s}$ is large enough). Formally, $p_{w}=$ $\max \left\{q_{w}-q_{s}+p_{s}, c_{w}\right\}, p_{s}=\max \left\{\frac{q_{s}+c_{w}+c_{s}}{2}, q_{s}-1+c_{w}\right\}$.

\section{Results}

Using the above strategies we compute, for the four scenarios, the implied expected utility (gross of the weak supplier's cost ${ }^{3}$ ) for the buyer as a function of $\Delta$, the cost difference between the weak and the strong bidder. The results are gathered in Figure 1. The main finding of the paper ${ }^{4}$ is that in each scenarios the best policy is $P U$ up to a threshold for $\Delta$ and $A S_{w}$ past the threshold. This means that revealing information about the buyer's preferences to the weak supplier is optimal whenever the cost disadvantage is large enough: levelling the field forces more competition from the strong. Conversely when the two bidders

\footnotetext{
${ }^{3}$ This is because a given value for $\Delta$ may result from different combinations of $c_{s}$ and $c_{w}$ We prevent this potential source of ambiguity by adding $c_{w}$ to buyer's expected utility.

${ }^{4}$ Details of the results underlying Figure 1 are available as online supplementary material to his paper.
} 
are similar public revelation is best. Further notice that the threshold is considerably smaller when the weak's characteristics are public than when they are not (whether or not the strong's are too). There is thus a significant difference between the all-public and all-private characteristics cases: under $P U$ the former case corresponds to an open-bid auction, while the latter is equivalent to a standard sealed-bid asymmetric auction. It is well known (see Maskin and Riley [9]) that in such contexts the weak bidder is more aggressive (and is better off) with the sealed-bid format: therefore comparatively small cost differences make it beneficial to "help" the weak when characteristics are public (as compared to when they are private).

\section{Acknowledgements}

We wish to thank Domenico Menicucci for useful comments. The usual disclaimer applies. This paper is part of the research project 2008RHJCKP-001 on Mechanism Design and Auctions wihich is financially supported by the Italian Ministry of the University. 


\section{References}

[1] Colucci, D., Doni, N., Valori, V., 2011. Information Disclosure in Procurement Auctions with Horizontally Differentiated Suppliers. DiMaD Working Papers 2011-02, Dipartimento di Matematica per le Decisioni, Universita' degli Studi di Firenze.

[2] Corns, A., Schotter, A., 1999. Can Affirmative Action Be Cost Effective? An Experimental Examination of Price-Preference Auctions. The American Economic Review 89, 291-305.

[3] Gal-Or, E., Gal-Or, M., Dukes, A., 2007. Optimal Information Revelation in Procurement Schemes. RAND Journal of Economics 38, 400-418.

[4] Hubbard, T.P., Paarsch, H.J., 2009. Investigating bid preferences at lowprice, sealed-bid auctions with endogenous participation. International Journal of Industrial Organization 27, 1-14.

[5] Kaplan, T.R., Zamir, S., Asymmetric First-Price Auctions with Uniform Distributions: Analytic Solutions to the General Case. Economic Theory, DOI: 10.1007/s00199-010-0563-9, (forthcoming).

[6] Kim, I., 1997. Government procurement and asymmetric rebate auctions. Economics Letters 54, 245-250.

[7] Lee, J., 2008. Favoritism in asymmetric procurement auctions. International Journal of Industrial Organization 26, 1407-1424.

[8] Li, H., Riley, J.G., 2007. Auction Choice. International Journal of Industrial Organization 25, 1269-1298.

[9] Maskin, E., Riley, J.G., 2000. Asymmetric Auctions. Review of Economic Studies 67, 413-438.

[10] McAfee, R.P., McMillan, J., 1989. Government Procurement and International Trade. Journal of Industrial Economics 26, 291-308.

[11] Rothkopf, M.H., Harstad, R.M., Fu, Y., 2003. Is Subsidizing Inefficient Bidders Actually Costly? Management Science 49, 71-84. 


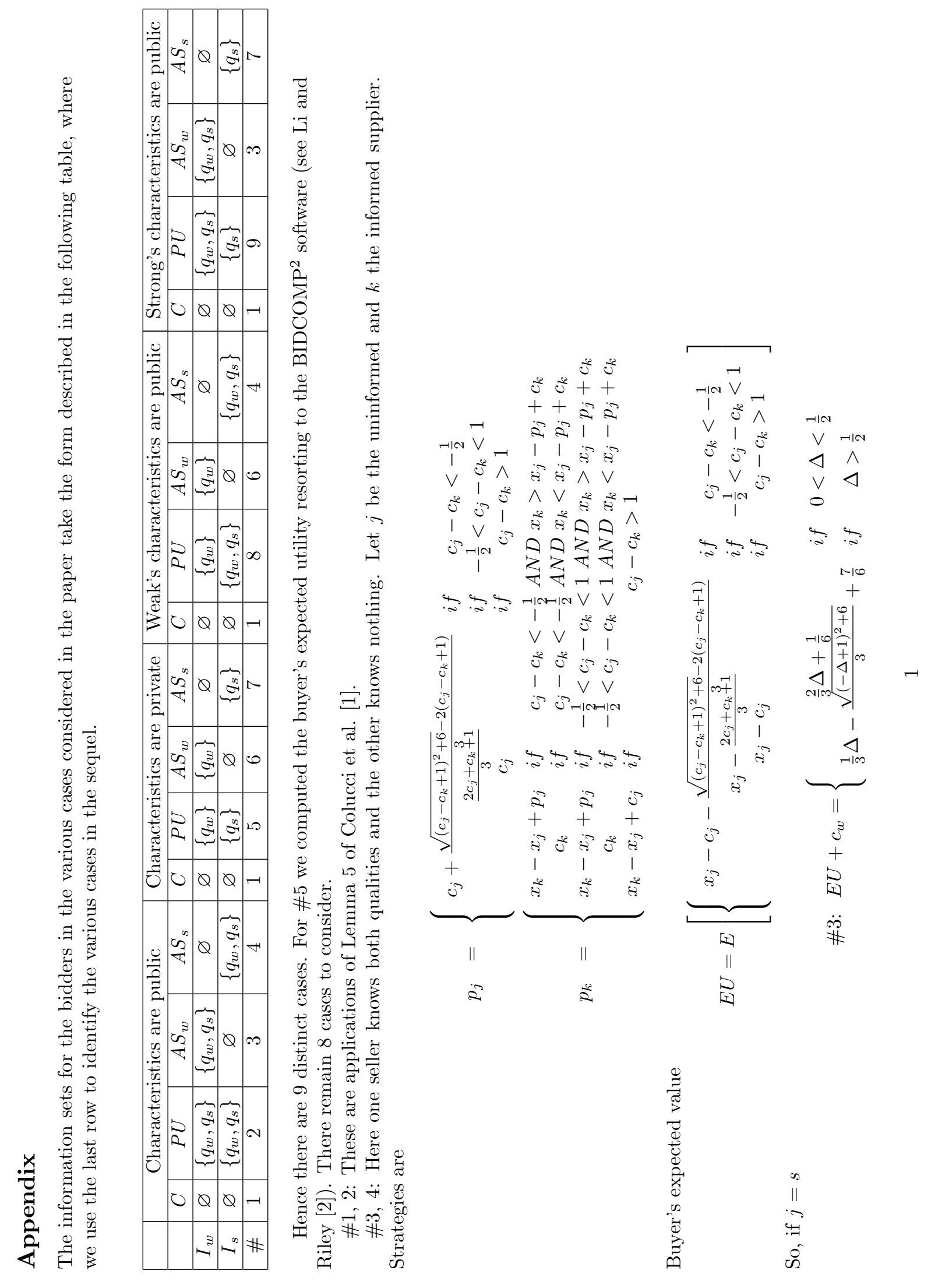




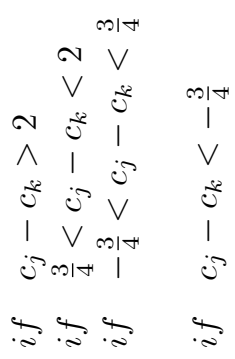

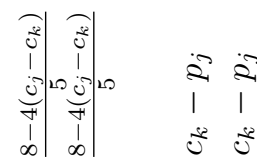

$\wedge V$ ला4 $\wedge V$

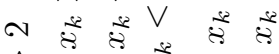

$\wedge \Omega 0$

ن

in N $V$ mमm

$\mathrm{V} \vee \operatorname{l|} \mid$

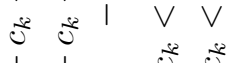

$1 \quad 1$ ग

$\begin{array}{llll}\mathrm{U} & \mathrm{V} & \mathrm{u} & \mathrm{u}\end{array}$

m|+m|t

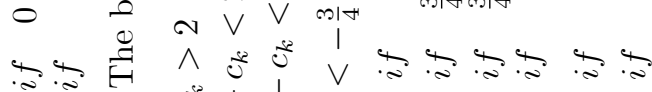

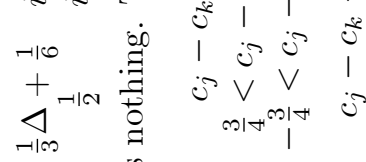

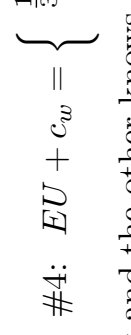

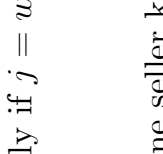

焉

$\ddot{\sim}$

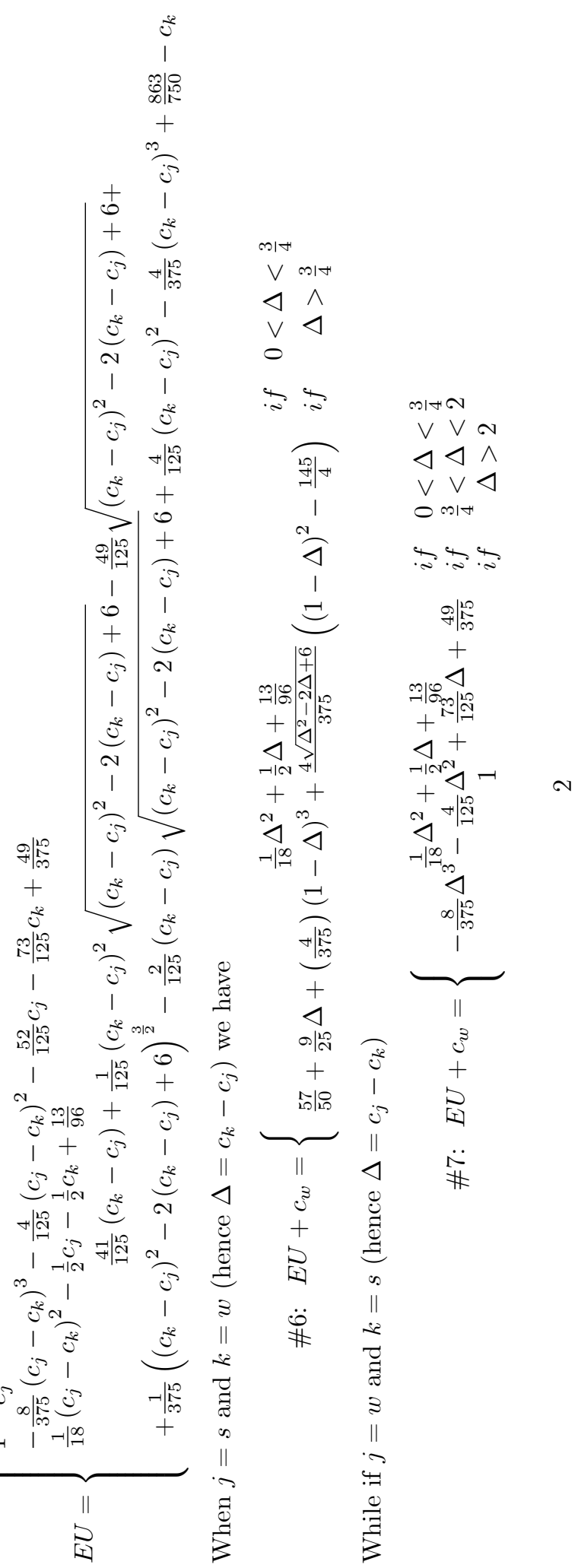




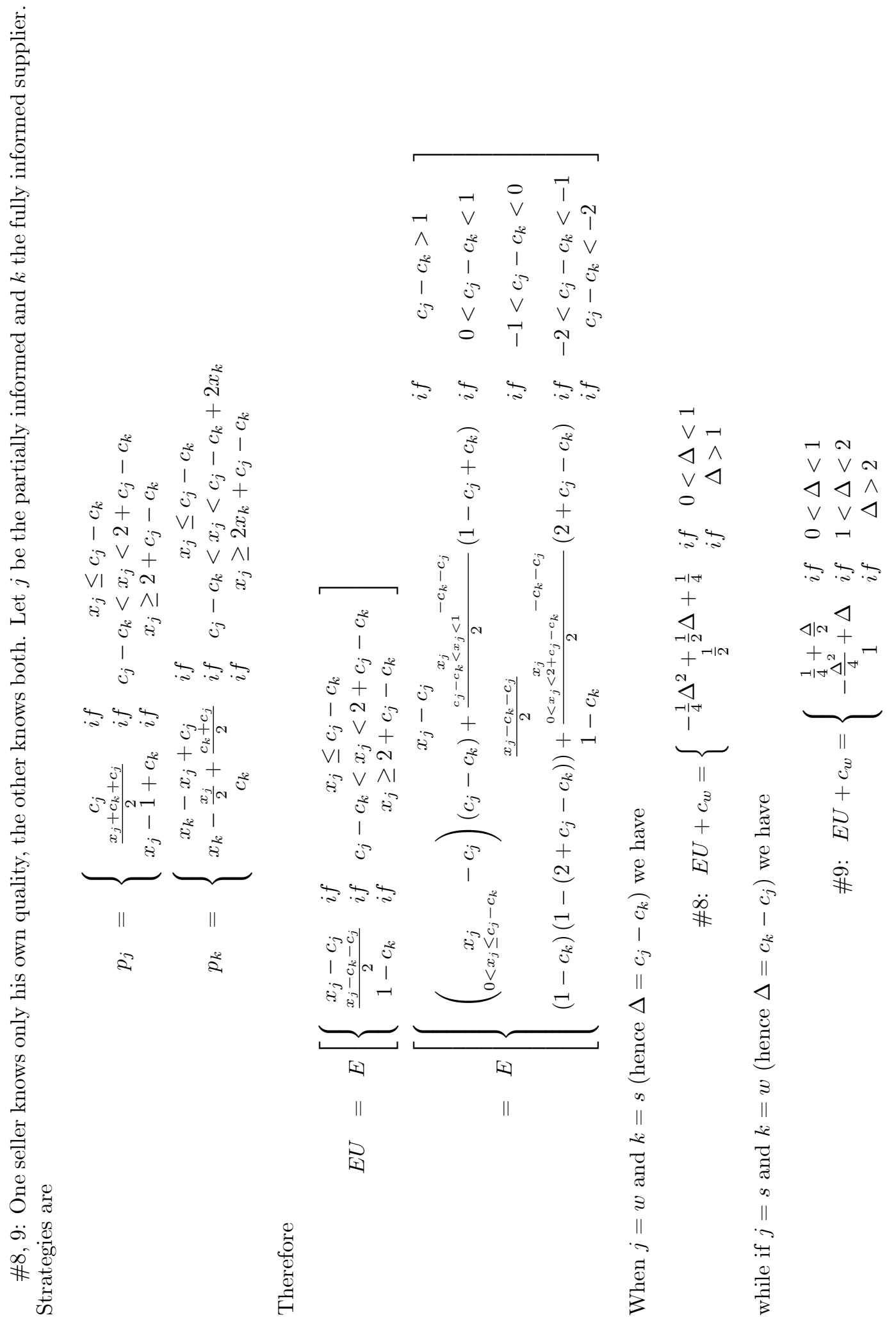

